\title{
How elite controllers and posttreatment controllers inform our search for an HIV-1 cure
}

\author{
Jonathan Z. Li' and Joel N. Blankson² \\ 'Division of Infectious Diseases, Brigham and Women's Hospital, Harvard Medical School, Boston, Massachusetts, USA. ²Center for AIDS Research, Department of Medicine, Johns Hopkins University, \\ Baltimore, Maryland, USA.

\begin{abstract}
A small percentage of people living with HIV-1 can control viral replication without antiretroviral therapy (ART). These patients are called elite controllers (ECs) if they are able to maintain viral suppression without initiating ART and posttreatment controllers (PTCs) if they control HIV replication after ART has been discontinued. Both types of controllers may serve as a model of a functional cure for HIV-1 but the mechanisms responsible for viral control have not been fully elucidated. In this review, we highlight key lessons that have been learned so far in the study of ECs and PTCs and their implications for HIV cure research.
\end{abstract}

Since the early days of the HIV epidemic, unusual individuals were reported who were able to suppress plasma viremia to very low or undetectable levels and maintain their $\mathrm{CD} 4^{+} \mathrm{T}$ cell counts for a prolonged period of time without antiretroviral therapy (ART) $(1,2)$. Over the years, these individuals have been known by a number of names, including HIV controllers, spontaneous controllers, elite controllers (ECs), elite suppressors, long-term nonprogressors, long-term asymptomatic, and long-term survivors, depending on the clinical and laboratory definition. Not long after combination ART became widely available, an intriguing letter was published regarding an individual in whom ART was initiated during early HIV infection with multiple viral loads greater than 80,000 HIV-1 RNA copies/mL (known as the first "Berlin patient"). The patient elected to stop treatment and, surprisingly, was able to sustain HIV suppression (3), in contrast to the rapid rebound reported in most individuals after ART discontinuation (4). While it is unclear how this individual would have fared without ART (5), this case represented the first report regarding a group of individuals now known as HIV posttreatment controllers (PTCs), individuals who are able to maintain HIV suppression despite stopping ART. There is some variability between studies in the minimum length of time that individuals must remain off ART, with some requiring 24 or more weeks of viral suppression (6) and others requiring 24 or more months (7). Since noncontrollers will experience rapid viral rebound within a median of 3-4 weeks after treatment interruption (4), these definitions are all expected to adequately capture this rare group of PTCs. While HIV ECs are rare, PTCs have been even more difficult to identify and study, largely because it is recommended that patients stay on ART lifelong and these individuals have largely been identified only as part of analytic treatment interruption (ATI) trials. While there may be some overlap between ECs and PTCs, there is increasing evidence that a substantial fraction of PTCs might have been unable to

Conflict of interest: JZL has consulted for Abbvie and JanBiotech.

Copyright: (c) 2021, American Society for Clinical Investigation.

Reference information: J Clin Invest. 2021;131(11):e149414.

https://doi.org/10.1172/JCl149414. achieve posttreatment control without the initial ART. Together, ECs and PTCs have already provided important insights about the mechanisms behind HIV remission. In this Review, we highlight five key lessons that have been learned so far and how they inform our search for an HIV cure.

\section{ART-free control of replication-competent virus is possible}

The existence of HIV ECs has given hope to the field and provided clear evidence that HIV can be controlled without the use of medications and that ART-free HIV remission is possible. In order to recognize controllers as legitimate models of a functional cure for HIV-1, these patients have to be controlling fully pathogenic virus. While there are studies suggesting that patients infected with attenuated HIV variants are more likely to become HIV controllers or long-term nonprogressors (8), there are also several lines of evidence to suggest that some controllers are infected with replication-competent virus (9). Multiple laboratories have cultured virus from a subset of these patients (10-14), and full genome sequence analysis of virus isolated from some controllers has not revealed any large deletions or signature mutations (10). There have also been several transmission pair studies in which transmission of virus between patients with progressive disease and HIV controllers has been documented (15-19). In cases in which replication-competent virus was isolated from both partners, comparable viral fitness was seen in three out of four of these pairs (15-17). There has also been documentation of evolution of plasma virus in cohorts of ECs (20-22). In some cases, this evolution has been in response to selective pressure by $\mathrm{CD}^{+} \mathrm{T}$ cells $(20,21)$. In another study, replication-competent viral isolates from ECs were shown to replicate vigorously and cause substantial $\mathrm{CD} 4^{+} \mathrm{T}$ cell depletion in humanized mice (14). Finally, reports of transient (23) or permanent loss of virologic control in ECs (24-27) indicate that the virus they were infected with was in fact replication competent.

There is evidence that PTCs also harbor HIV with preserved viral fitness. This evidence includes data reported from two of the more comprehensive studies to date on HIV PTCs: the French VISCONTI study of 14 early-treated individuals (7) and the Con- 
trol of HIV after Antiretroviral Medication Pause (CHAMP) study of 67 PTCs from a consortium of North American studies (6). First, in both of these studies and others (28), PTCs were found to have high levels of pre-ART viremia that were similar to levels found in posttreatment noncontrollers (NCs). In the VISCONTI study, levels of pre-ART viral load were markedly higher in the PTCs compared with the viral loads of HIV ECs during acute infection. Second, a substantial subset of CHAMP PTCs were found to have early viral load rebound before regaining control, including $33 \%$ of individuals who had early viral rebound peaks above 10,000 HIV-1 RNA copies/mL. Third, robust HIV production could be induced from ex vivo stimulated cells of PTCs from the VISCONTI study (7) and other studies $(6,29-32)$. Fourth, the analysis of transmission pairs has also shown that the same HIV viral strain infecting a PTC has robust replication kinetics (33) and can lead to endstage HIV in a partner (30). Fifth, posttreatment control can be maintained despite an onslaught of viral pressure, including evidence of HIV evolution, dual infection, and even superinfection (34). Finally, eventual loss of viral control has been documented in a subset of PTCs, confirming the pathogenicity of the infecting viruses $(6,35)$. Together, these results demonstrate that an attenuated strain of HIV is not the primary mechanism behind HIV elite and posttreatment control and affirm that these patients represent important models of sustained HIV remission.

\section{HIV controllers have unusual reservoir characteristics}

Newer assays enable investigators to better characterize the reservoir and distinguish between intact provirus and those with large deletions and/or hypermutations (36-38). Using the intact proviral DNA assay (38), Kwaa et al. reported that the frequency of intact HIV DNA was about 20-fold lower than the frequency seen in people living with HIV (PLWH) on ART (39). Jiang and colleagues performed full proviral genome sequencing on large numbers of ECs and chronic progressors (CPs) and also found much lower levels of intact DNA in elites. Interestingly, one EC had a single intact proviral clone detected from 1.02 billion $\mathrm{CD} 4^{+}$ $\mathrm{T}$ cells, and another $\mathrm{EC}$ had no proviral clones detected from 1.5 billion $\mathrm{CD} 4^{+} \mathrm{T}$ cells (40). This study is the best characterization of a subset of ECs called exceptional elite controllers (EECs) who appear to have smaller reservoirs than the majority of ECs (4144). Further studies will be needed to determine whether these patients have in fact achieved a sterilizing cure. Several studies have looked at integration sites in HIV controllers and provided evidence of clonal expansion in these participants $(31,40,45)$. Studies looking at total and intact proviral DNA found that the frequency of clonally expanded clones in ECs exceeds the frequency seen in PLWH on ART $(31,40)$ and that the percentage of intact proviral clones that were integrated into non-genic or pseudogenic regions was higher in ECs than in PLWH on ART (40). There was also more enrichment of integration sites in satellite DNA and genes in the zinc finger family in ECs compared with CPs (40). These integration sites would be more likely to result in transcriptional repression, and in fact there was evidence of a lower ratio of viral transcripts to viral DNA. It should be emphasized that these characteristics are more likely a consequence rather than a cause of elite control.
PTCs also have unusual features related to both the size of the reservoir and distribution within $\mathrm{T}$ cell subsets. In a study of ten PTCs identified from AIDS Clinical Trials Group (ACTG) ATI trials, PTCs had a restricted reservoir size, featuring an approximately 7 -fold smaller total and intact proviral reservoir compared with NCs, although the proportion of intact proviruses was not significantly different between PTCs and NCs (29). As with ARTsuppressed individuals, clonally expanded populations of HIVinfected cells appear in PTCs, and likely at a frequency similar to that in NCs (29). The VISCONTI study also reported that PTCs had low levels of HIV DNA that continued to decline off ART (7). Interestingly, the VISCONTI study and others (32) described differences in the reservoir distribution between $\mathrm{T}$ cell subsets, with PTCs having smaller reservoirs in the longer-lived naive and central memory populations. Similarly to the EECs, PTCs have also been reported with extremely low levels of HIV reservoirs, which included sampling from tissue compartments (46). However, it is important to note that detectable HIV DNA and cell-associated RNA do not preclude the possibility of posttreatment control (7, 28-30). Finally, nonhuman primate (NHP) studies have also reported limited SIV reservoir size in animals able to maintain viral control after treatment interruption (47). The evidence that HIV reservoirs in HIV controllers are severely restricted in size and demonstrate features of deep latency in ECs is intriguing. These findings highlight the potency of antiviral responses in HIV controllers, but also that transcriptional silencing of HIV proviruses may play a role in long-term viral control. Additional studies in PTCs of HIV reservoir maintenance and transcriptional regulation are needed, including studies of proviral integration sites.

\section{Early ART or immune response is important for control}

The few studies of ECs during primary infection suggest that these individuals do not have the high levels of viremia seen in patients with progressive disease (48). Furthermore, these ECs appear to achieve virologic control very early in the course of infection (49, 50). It is almost unheard of for a person who is viremic more than a year after primary infection to subsequently control viral replication without ART.

Since the description of the original acutely treated "Berlin patient," the majority of reports have profiled pediatric or adult patients who initiated ART during early infection $(3,7,30,32,33$, 51-55). This phenomenon does not appear to be restricted to earlytreated individuals, as there are cases of PTCs who initiated ART during chronic infection $(56,57)$, including an individual who had previously progressed to AIDS (46). The largest study to date comparing the frequency of posttreatment control in those initiating ART during early versus chronic infection was the CHAMP study, which confirmed that early treatment was associated with a greater frequency of posttreatment control with $13 \%$ of early-treated versus $4 \%$ of chronic-treated participants meeting the study's definition of posttreatment control (6). Several areas of uncertainty remain, including the timing of early ART initiation, as the majority of PTCs were treated during Fiebig stages III to V of infection while studies of individuals who initiated ART during the earliest Fiebig stages had rapid rebound after ATI (58). These reports are also bolstered by NHP studies of early antibody treatment that 
induced posttreatment control $(59,60)$. Early suppression of viral replication likely benefits individuals in multiple ways. First, early ART initiation substantially restricts the size of the HIV reservoir (61) with greater than 100-fold difference in the frequency of cells harboring HIV DNA 3 years after infection (62). In addition, early ART initiation is likely to have long-term benefits for preserving functional cellular immune responses to $\operatorname{HIV}(63,64)$. These results provide additional support for early initiation of ART for all PLWH and suggest that early-treated individuals have a lower barrier to HIV remission and may be ideal candidates for HIV curative studies.

\section{Sex and genetics matter ... kind of}

Recent studies highlight important sex differences in HIV pathogenesis (reviewed in ref. 65) and indicate that females are more likely than males to achieve controller status in both adult $(66,67)$ and pediatric (68) cohorts. Interestingly, the female partner has been the controller in all four transmission pairs we have studied (15-17). HLA-B ${ }^{\star} 57$ and HLA-B ${ }^{\star} 27$ were overrepresented in ECs in both cohort studies $(69,70)$ and genome-wide association studies $(71,72)$. However, these alleles alone incompletely explain elite control, as they are also present in many CPs. In order to define additional factors that may modulate elite control, Martin et al. performed whole human genome sequencing on large numbers of HLA-B ${ }^{\star} 57$ ECs and HLA-B ${ }^{\star} 57$ CPs and identified a single variant, present in the NK cell receptor KIR3DL1, as the only notable difference between the two cohorts of patients (73). However, this variant does not fully explain why the majority of patients with protective HLA alleles do not become ECs.

It has been challenging to translate the favorable host genetics of ECs into broadly applicable therapies. So it was particularly interesting that the VISCONTI, ACTG, and many other studies did not report an increased frequency of protective HLA alleles in PTCs $(7,29,30,33,53,74)$. This lack of protective gene candidates raises the possibility that posttreatment control mechanisms may differ between ECs and PTCs and that the lessons learned from PTCs may translate better into treatment strategies. The role that sex and genetics play in posttreatment control has also been highlighted in two studies of African participants. The first study analyzed participants of the PROMISE trial, which enrolled virologically suppressed postpartum women, the majority of whom were from Africa. Interestingly, $25 \%$ of women in the PROMISE study were virologically suppressed 12 weeks after ATI compared with only $6 \%$ of ACTG participants, who were predominantly male and from the United States (75). A second study found a greater probability of posttreatment control in female participants in the SPARTAC study who were African versus those who were nonAfrican (76). However, neither study was able to fully differentiate between the potential effects of sex, race, and host or viral genetics. Additional studies are needed to determine whether EC and PTC prevalence differs between men and women, and between individuals infected with different HIV subtypes (77).

\section{Immune mediators may differ between ECs and PTCs}

The mechanisms responsible for HIV replication control in ECs are still not fully understood, and it has been challenging to dis- tinguish between cause and consequence of control. In fact, most studies are not performed during primary infection while control is being achieved. As described above, the most objective data suggest that class I HLA alleles are associated with protection. Since class I HLA proteins present peptides to $\mathrm{CD}^{+} \mathrm{T}$ cells, it stands to reason that $\mathrm{CD} 8^{+} \mathrm{T}$ cells play a key role in control. Indirect evidence for the role of $\mathrm{CD}^{+} \mathrm{T}$ cells comes from the monkey model of elite control, in which protective MHC class I alleles have also been described (78). Depletion of $\mathrm{CD}^{+} \mathrm{T}$ cells in EC monkeys results in transient loss of control, suggesting that these cells are playing a role in suppressing viral replication $(79,80)$. In humans, there is strong evidence that $\mathrm{CD} 8^{+} \mathrm{T}$ cells from ECs are much better at controlling viral replication in vitro than $\mathrm{CD}^{+} \mathrm{T}$ cells from PLWH on ART (31, 81-85). This enhanced control has been associated with distinct $\mathrm{CD}^{+} \mathrm{T}$ cell metabolic (86-89) and transcriptional (90-92) profiles. Not all ECs have protective HLA alleles, but one study suggested that many of these ECs have HIV-specific $\mathrm{CD}^{+} \mathrm{T}$ cells that kill as effectively as HIV-specific $\mathrm{CD} 8^{+} \mathrm{T}$ cells in ECs with protective HLA alleles (85). This cytotoxic capacity may be due to the fact that $\mathrm{CD} 8^{+} \mathrm{T}$ cells from controllers with and without protective HLA alleles target highly constrained epitopes that do not tolerate mutations $(93,94)$. While peripheral $\mathrm{CD}^{+} \mathrm{T}$ cells can control viral replication by killing infected $\mathrm{CD} 4^{+} \mathrm{T}$ cells, recent studies suggest that $\mathrm{CD} 8^{+} \mathrm{T}$ cells from lymph nodes do not have robust cytolytic function (95) and are phenotypically similar to tissue-resident memory CD8 ${ }^{+} \mathrm{T}$ cells (96). Interestingly, ECs have higher levels of HIV-specific tissue-resident memory $\mathrm{CD}^{+}$ $\mathrm{T}$ cells than CPs in secondary lymphoid organs, suggesting that these cells control viral replication in the occupied tissue $(96,97)$. ECs with weak CD8 ${ }^{+} \mathrm{T}$ cell responses have been noted (98), but a study suggests that these subjects still possess strong HIV-specific central memory responses (99).

There is evidence that HIV-specific T cells can play a role in determining viral set point after treatment interruption (100). Early ART can preserve polyfunctional T cell responses in PTCs (101), and some harbor CD8 ${ }^{+} \mathrm{T}$ cells with the ex vivo capacity to suppress HIV replication (31). In addition, depletion of $\mathrm{CD}^{+} \mathrm{T}$ cells in NHP models of antibody-mediated posttreatment control have led to viral rebound (60). However, the immune mediators of HIV posttreatment control remain unclear, as most of the available data in PTCs have demonstrated relatively modest HIV-specific T cell responses $(7,28,30,32,33,46,52,55)$. In the patient known as the "Mississippi child," HIV-specific T cell and antibody responses were largely undetectable during the 21.9 months of remission and returned only at the time of viremic rebound (35). The immune mediators of posttreatment control remain an enduring mystery, and a priority area of research.

The viral control demonstrated in ECs is not without cost, as ECs show increased $\mathrm{T}$ cell activation and inflammation (102-104). The impact of sustained immune activation and inflammation in noncontrollers has been associated with poor clinical outcomes (105-108) and extends to ECs, who may have an increased risk of cardiovascular disease (109) and hospitalization (110), although the risk remains undetermined in some studies (111-113). Intriguingly, PTCs can maintain viral control despite generally exhibiting blunted levels of immune activation compared with ECs (7, $28,32,33,55,99)$, but additional studies are needed to assess the 

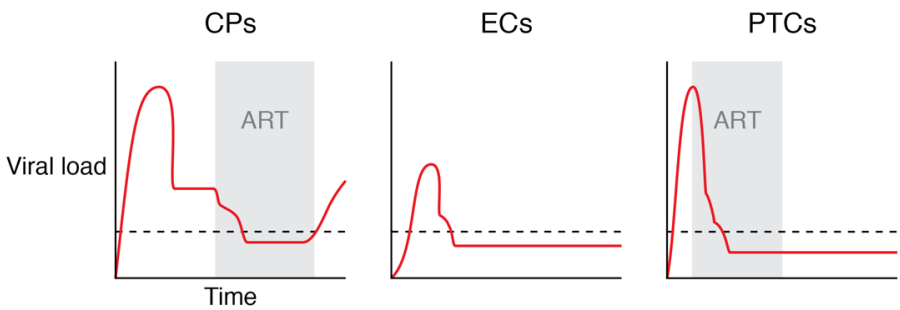

Prevalence*

$>80 \%$

$\%$ with protective HLA alleles

$10 \%-20 \%$

HIV-specific CD8 ${ }^{+} \mathrm{T}$ cell response

Reservoir size

Clonally expanded latent cells

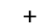

$+++$

$++$

Figure 1. Virologic and immunologic profiles of CPs, ECs, and PTCs. ART is normally started in chronic progressors (CPs) during the chronic phase of infection, and a rebound in viremia is seen when therapy is discontinued. In contrast, elite controllers (ECs) are ART-naive subjects who control viral replication naturally. Posttreatment controllers (PTCs) are more often patients in whom ART is initiated during primary infection. These patients maintain control of viral replication when ART is discontinued. * Estimates depend on definition of EC and PTC.,+++ , and +++ indicate relative magnitude of each parameter.

long-term clinical implications of HIV posttreatment control and whether patients can achieve HIV remission without increased risk of systemic inflammation and clinical events.

\section{Concluding remarks}

The study of medical outliers with unusual disease phenotypes or responses to treatment has led to important medical advances. In the oncology field, research on exceptional responders to cancer therapeutics has led to a deeper understanding of the molecular basis of treatment response and helped revolutionize the personalized medicine approach to the selection of treatment regimens $(114,115)$. Similarly, ECs and PTCs represent outliers in HIV natural infection and treatment outcomes (Figure 1). These individuals can maintain HIV suppression of pathogenic, replication-competent virus and serve as models of a functional cure for HIV. Studies of these patients have already led to important insight into the natural control of HIV, and the expe- $\sim 13 \%-15 \%$ (early-treated)

$10 \%-20 \%$

$+$

$++$

$++$ jblanks@jhmi.edu.

rience with ECs suggests that combining ART, initiated during primary infection, with a therapeutic vaccination strategy that induces potent $\mathrm{CD} 8^{+} \mathrm{T}$ cell responses targeting conserved epitopes may be a viable strategy. While protective HLA alleles are overrepresented in ECs, it is reassuring that these alleles are not necessary to achieve long-term remission. An enhanced immune response may lead to HIV remission not only by the clearance of activated HIV-infected cells, but also potentially by selecting for HIV-infected cells that are in a deeper state of HIV latency. Studies of PTCs have highlighted the importance of initiating ART during primary infection, as we may be able to induce posttreatment control in more than $10 \%$ of patients $(27$, 28). The low levels of immune activation and systemic inflammation detected in PTCs represent a hopeful sign that we may be able to induce HIV remission without the clinical risks observed in some HIV ECs. However, PTCs remain understudied as a group, and a concerted international effort is needed to identify these individuals and uncover the mechanisms behind their ability to achieve sustained HIV remission.

\section{Acknowledgments}

JNB is supported by the Johns Hopkins University Center for AIDS Research (P30AI094189) and National Institute of Allergy and Infectious Diseases (NIAID) award R01AI120024. JZL is supported by NIAID award R01AI150396.

Address correspondence to: Jonathan Z. Li, Brigham and Women's Hospital, Harvard Medical School, 65 Landsdowne Street, Room 421, Cambridge, Massachusetts 02139, USA. Phone: 617.768.8476; Email: jli@bwh.harvard.edu. Or to: Joel N. Blankson, Johns Hopkins University School of Medicine, 733 North Broadway, Baltimore, Maryland 21205, USA. Phone: 443.676.9951; Email:

1. Cao Y, et al. Virologic and immunologic characterization of long-term survivors of human immunodeficiency virus type 1 infection. $N$ EnglJ Med.1995;332(4):201-208.

2. Hogervorst E, et al. Predictors for non- and slow progression in human immunodeficiency virus (HIV) type 1 infection: low viral RNA copy numbers in serum and maintenance of high HIV-1 p24-specific but not V3-specific antibody levels. J Infect Dis. 1995;171(4):811-821.

3. Lisziewicz J, et al. Control of HIV despite the discontinuation of antiretroviral therapy. $N$ Engl JMed.1999;340(21):1683-1684.

4. Li JZ, et al. The size of the expressed HIV reservoir predicts timing of viral rebound after treatment interruption. AIDS. 2016;30(3):343-353.

5. Jessen $\mathrm{H}$, et al. How a single patient influenced
HIV research-15-year follow-up. $N$ Engl J Med. 2014;370(7):682-683.

6. Namazi G, et al. The Control of HIV After Antiretroviral Medication Pause (CHAMP) study: posttreatment controllers identified from 14 clinical studies. J Infect Dis. 2018;218(12):1954-1963.

7. Saez-Cirion A, et al. Post-treatment HIV-1 controllers with a long-term virological remission after the interruption of early initiated antiretroviral therapy ANRS VISCONTI Study. PLoS Pathog. 2013;9(3):e1003211.

8. Zaunders J, et al. The Sydney Blood Bank Cohort: implications for viral fitness as a cause of elite control. Curr Opin HIV AIDS. 2011;6(3):151-156.

9. Woldemeskel BA, et al. Viral reservoirs in elite controllers of HIV-1 infection: implications for HIV cure strategies. EBioMedicine.
2020;62:103118.

10. Blankson JN, et al. Isolation and characterization of replication-competent human immunodeficiency virus type 1 from a subset of elite suppressors. J Virol. 2007;81(5):2508-2518.

11. Lamine A, et al. Replication-competent HIV strains infect HIV controllers despite undetectable viremia (ANRS EP36 study). AIDS. 2007;21(8):1043-1045.

12. Julg B, et al. Infrequent recovery of HIV from but robust exogenous infection of activated CD4(+) T cells in HIV elite controllers. Clin Infect Dis. 2010;51(2):233-238.

13. Chun TW, et al. Effect of antiretroviral therapy on HIV reservoirs in elite controllers. J Infect Dis. 2013;208(9):1443-1447.

14. Salgado M, et al. HLA-B ${ }^{\star} 57$ elite suppressor and 
chronic progressor HIV-1 isolates replicate vigorously and cause $\mathrm{CD} 4^{+} \mathrm{T}$ cell depletion in humanized BLT mice. JVirol. 2014;88(6):3340-3352.

15. Bailey JR, et al. Transmission of human immunodeficiency virus type 1 from a patient who developed AIDS to an elite suppressor. J Virol. 2008;82(15):7395-7410.

16. Buckheit RW 3rd, et al. Host factors dictate control of viral replication in two HIV-1 controller/ chronic progressor transmission pairs. Nat Commun. 2012;3:716.

17. Walker-Sperling VE, et al. Factors associated with the control of viral replication and virologic breakthrough in a recently infected HIV-1 controller. EBioMedicine. 2017;16:141-149.

18. Yue L, et al. Transmitted virus fitness and host $T$ cell responses collectively define divergent infection outcomes in two HIV-1 recipients. PLoS Pathog. 2015;11(1):e1004565.

19. Bendenoun M, et al. What is the most important for elite control: genetic background of patient, genetic background of partner, both or neither? Description of complete natural history within a couple of MSM. EBioMedicine. 2018;27:51-60.

20. Bailey JR, et al. Maintenance of viral suppression in HIV-1-infected HLA-B ${ }^{*} 7^{+}$elite suppressors despite CTL escape mutations. J Exp Med. 2006;203(5):1357-1369.

21. O'Connell KA, et al. Control of HIV-1 in elite suppressors despite ongoing replication and evolution in plasma virus. J Virol. 2010;84(14):7018-7028.

22. Mens $\mathrm{H}$, et al. HIV-1 continues to replicate and evolve in patients with natural control of HIV infection. JVirol. 2010;84(24):12971-12981.

23. Smith NM, et al. Proof-of-principle for immune control of global HIV-1 reactivation in vivo. Clin Infect Dis. 2015;61(1):120-128.

24. Bailey JR, et al.Evolution of HIV-1 in an HLA-B ${ }^{*} 57$-positive patient during virologic escape. J Infect Dis. 2007;196(1):50-55.

25. Rosás-Umbert M, et al. Mechanisms of abrupt loss of virus control in a cohort of previous HIV controllers. J Virol. 2019;93(4):e01436-18.

26. Leon $\mathrm{A}$, et al. Rate and predictors of progression in elite and viremic HIV-1 controllers. AIDS. 2016;30(8):1209-1220.

27. Borrell M, et al. High rates of long-term progression in HIV-1-positive elite controllers. J Int AIDS Soc. 2021;24(2):e25675.

28. Frange P, et al. HIV-1 virological remission lasting more than 12 years after interruption of early antiretroviral therapy in a perinatally infected teenager enrolled in the French ANRS EPFCO10 paediatric cohort: a case report. Lancet HIV. 2016;3(1):e49-e54.

29. Sharaf R, et al. HIV-1 proviral landscapes distinguish posttreatment controllers from noncontrollers. JClin Invest. 2018;128(9):4074-4085.

30. Salgado M, et al. Prolonged control of replication-competent dual- tropic human immunodeficiency virus-1 following cessation of highly active antiretroviral therapy. Retrovirology. 2011;8:97.

31. Veenhuis RT, et al. Long-term remission despite clonal expansion of replication-competent HIV-1 isolates. JCI Insight. 2018;3(18):e122795.

32. Chéret A, et al. Combined ART started during acute HIV infection protects central memory
$\mathrm{CD}^{+} \mathrm{T}$ cells and can induce remission. JAntimicrob Chemother. 2015;70(7):2108-2120.

33. Persaud D, et al. Absence of detectable HIV-1 viremia after treatment cessation in an infant. N Engl J Med. 2013;369(19):1828-1835.

34. Etemad B, et al. Final Program. Paper presented at: 9th Edition: HIV Persistence During Therapy: Reservoirs \& Eradication Strategies Workshop; December 10-13, 2019; Miami, Florida, USA. https://www.hiv-persistence.com/program-final. Accessed April 13, 2021.

35. Luzuriaga K, et al. Viremic relapse after HIV-1 remission in a perinatally infected child. $N$ Engl J Med. 2015;372(8):786-788.

36. Ho YC, et al. Replication-competent noninduced proviruses in the latent reservoir increase barrier to HIV-1 cure. Cell. 2013;155(3):540-551.

37. Einkauf KB, et al. Intact HIV-1 proviruses accumulate at distinct chromosomal positions during prolonged antiretroviral therapy. J Clin Invest. 2019;129(3):988-998.

38. Bruner KM, et al. A quantitative approach for measuring the reservoir of latent HIV-1 proviruses. Nature. 2019;566(7742):120-125.

39. Kwaa AK, et al. Elite suppressors have low frequencies of intact HIV-1 proviral DNA. AIDS. 2020;34(4):641-643.

40. Jiang C, et al. Distinct viral reservoirs in individuals with spontaneous control of HIV-1. Nature. 2020;585(7824):261-267.

41. Mendoza D, et al. Comprehensive analysis of unique cases with extraordinary control over HIV replication. Blood. 2012;119(20):4645-4655.

42. Canouï E, et al. A subset of extreme human immunodeficiency virus (HIV) controllers is characterized by a small HIV blood reservoir and a weak T-cell activation level. Open Forum Infect Dis. 2017;4(2):ofx064.

43. Zaunders J, et al. Possible clearance of transfusion-acquired nef/LTR-deleted attenuated HIV-1 infection by an elite controller with CCR $5 \Delta 32$ heterozygous and HLA-B57 genotype. J Virus Erad. 2019;5(2):73-83.

44. Casado C, et al. Permanent control of HIV-1 pathogenesis in exceptional elite controllers: a model of spontaneous cure. Sci Rep. 2020;10(1):1902.

45. Boritz EA, et al. Multiple origins of virus persistence during natural control of HIV infection. Cell. 2016;166(4):1004-1015.

46. Uruena A, et al. Prolonged posttreatment virologic control and complete seroreversion after advanced human immunodeficiency virus-1 infection. Open Forum Infect Dis. 2021;8(1):ofaa613.

47. Strongin Z, et al. Virologic and immunologic features of simian immunodeficiency virus control post-ART interruption in rhesus macaques. J Virol. 2020;94(14):e00338-20.

48. Goujard C, et al. Spontaneous control of viral replication during primary HIV infection: when is "HIV controller" status established? Clin Infect Dis. 2009;49(6):982-986.

49. Walker-Sperling VE, et al. Factors associated with the control of viral replication and virologic breakthrough in a recently infected HIV-1 controller. EBioMedicine. 2017;16:141-149.

50. Morley D, et al. Rapid development of HIV elite control in a patient with acute infection. $B M C$ Infect Dis. 2019;19(1):815.

51. Hocqueloux L, et al. Long-term immunovirologic control following antiretroviral therapy interruption in patients treated at the time of primary HIV-1 infection. AIDS. 2010;24(10):1598-1601.

52. Goujard C, et al. HIV-1 control after transient antiretroviral treatment initiated in primary infection: role of patient characteristics and effect of therapy. Antivir Ther. 2012;17(6):1001-1009.

53. Maenza J, et al. How often does treatment of primary HIV lead to post-treatment control? Antivir Ther. 2015;20(8):855-863.

54. Martin GE, et al. Post-treatment control or treated controllers? Viral remission in treated and untreated primary HIV infection. AIDS. 2017;31(4):477-484.

55 . Violari A, et al. A child with perinatal HIV infection and long-term sustained virological control following antiretroviral treatment cessation. Nat Commun. 2019;10(1):412.

56. Maggiolo F, et al. Post treatment controllers after treatment interruption in chronically HIV infected patients. AIDS. 2018;32(5):623-628.

57. Perkins MJ, et al. Brief report: Prevalence of posttreatment controller phenotype is rare in HIV-infected persons after stopping antiretroviral therapy. J Acquir Immune Defic Syndr. 2017;75(3):364-369.

58. Colby DJ, et al. Rapid HIV RNA rebound after antiretroviral treatment interruption in persons durably suppressed in Fiebig I acute HIV infection. Nat Med. 2018;24(7):923-926.

59. Haigwood NL, et al. Passive immune globulin therapy in the SIV/macaque model: early intervention can alter disease profile. Immunol Lett. 1996;51(1-2):107-114.

60. Nishimura Y, et al. Early antibody therapy can induce long-lasting immunity to SHIV. Nature. 2017;543(7646):559-563.

61. Pinzone MR, et al. Monitoring integration over time supports a role for cytotoxic T lymphocytes and ongoing replication as determinants of reservoir size. J Virol. 2016;90(23):10436-10445.

62. Ananworanich J, et al. HIV DNA set point is rapidly established in acute HIV infection and dramatically reduced by early ART. EBioMedicine. 2016;11:68-72.

63. Trautmann L, et al. Profound metabolic, functional, and cytolytic differences characterize HIV-specific CD8 T cells in primary and chronic HIV infection. Blood. 2012;120(17):3466-3477.

64. Streeck $\mathrm{H}$, et al. Immunological and virological impact of highly active antiretroviral therapy initiated during acute HIV-1 infection. J Infect Dis. 2006;194(6):734-739.

65. Scully EP. Sex differences in HIV infection. Curr HIV/AIDS Rep. 2018;15(2):136-146.

66. Madec Y, et al. Spontaneous control of viral load and CD 4 cell count progression among HIV-1 seroconverters. AIDS. 2005;19(17):2001-2007.

67. Madec Y, et al. Natural history of HIV-control since seroconversion. AIDS. 2013;27(15):2451-2460.

68. Vieira VA, et al. Strong sex bias in elite control of paediatric HIV infection. AIDS. 2019;33(1):67-75.

69. Kaslow RA, et al. Influence of combinations of human major histocompatibility complex genes on the course of HIV-1 infection. Nat Med. 
1996;2(4):405-411.

70. Migueles SA, et al. HLA B ${ }^{\star} 5701$ is highly associated with restriction of virus replication in a subgroup of HIV-infected long term nonprogressors. Proc Natl Acad Sci US A. 2000;97(6):2709-2714.

71. Fellay J, et al. A whole-genome association study of major determinants for host control of HIV-1. Science. 2007;317(5840):944-947.

72. International HIV Controllers Study, et al. The major genetic determinants of HIV-1 control affect HLA class I peptide presentation. Science. 2010;330(6010):1551-1557.

73. Martin MP, et al. Killer cell immunoglobulinlike receptor 3DL1 variation modifies HLA$\mathrm{B}^{*} 57$ protection against HIV-1. J Clin Invest. 2018;128(5):1903-1912.

74. Etemad B, et al. Abstract 347. Paper presented at: Conference on Retroviruses and Opportunistic Infections (CROI 2016); February 22-25, 2016; Boston, Massachusetts, USA. https:/i-base.info/ htb/29879. Accessed April 13, 2021.

75. Le CN, et al. Time to viral rebound and safety after antiretroviral treatment interruption in postpartum women compared with men. AIDS. 2019;33(14):2149-2156.

76. Gossez M, et al. Virological remission after antiretroviral therapy interruption in female African HIV seroconverters. AIDS. 2019;33(2):185-197.

77. Dos Santos JS, et al. Host factor predictors in long-term nonprogressors HIV-1 infected with distinct viral clades. Curr HIV Res. 2017;15(6):440-447.

78. Mudd PA, Watkins DI. Understanding animal models of elite control: windows on effective immune responses against immunodeficiency viruses. Curr Opin HIV AIDS. 2011;6(3):197-201.

79. Friedrich TC, et al. Subdominant CD $8^{+} \mathrm{T}$-cell responses are involved in durable control of AIDS virus replication. J Virol. 2007;81(7):3465-3476.

80. Pandrea I, et al. Functional cure of SIVagm infection in rhesus macaques results in complete recovery of $\mathrm{CD}^{+} \mathrm{T}$ cells and is reverted by $\mathrm{CD} 8^{+}$ cell depletion. PLoS Pathog. 2011;7(8):e1002170.

81. Migueles SA, et al. HIV-specific CD8 ${ }^{+} \mathrm{T}$ cell proliferation is coupled to perforin expression and is maintained in nonprogressors. Nat Immunol. 2002;3(11):1061-1068.

82. Betts MR, et al HIV nonprogressors preferentially maintain highly functional HIV-specific $\mathrm{CD}^{+} \mathrm{T}$ cells. Blood. 2006;107(12):4781-4789.

83. Sáez-Cirión A, et al. HIV controllers exhibit potent CD8 $\mathrm{T}$ cell capacity to suppress HIV infection ex vivo and peculiar cytotoxic T lymphocyte activation phenotype. Proc Natl Acad Sci US A. 2007;104(16):6776-6781.

84. Migueles SA, et al. Lytic granule loading of CD8 ${ }^{+}$ $\mathrm{T}$ cells is required for HIV-infected cell elimination associated with immune control. Immunity. 2008;29(6):1009-1021.

85. Migueles SA, et al. CD8(+) T-cell cytotoxic capac- ity associated with human immunodeficiency virus- 1 control can be mediated through various epitopes and human leukocyte antigen types. EBioMedicine. 2014;2(1):46-58.

86. Chowdhury FZ, et al. Metabolic pathway activation distinguishes transcriptional signatures of $\mathrm{CD}^{+} \mathrm{T}$ cells from HIV-1 elite controllers. AIDS. 2018;32(18):2669-2677.

87. Tarancon-Diez L, et al. Immunometabolism is a key factor for the persistent spontaneous elite control of HIV-1 infection. EBioMedicine. 2019;42:86-96.

88. Angin M, et al. Metabolic plasticity of HIVspecific $\mathrm{CD}^{+} \mathrm{T}$ cells is associated with enhanced antiviral potential and natural control of HIV-1 infection. Nat Metab. 2019;1(7):704-716.

89. Loucif H, et al. Lipophagy confers a key metabolic advantage that ensures protective CD8A T-cell responses against HIV-1. Autophagy. 2021;18:1-16.

90. Simonetta F, et al. High eomesodermin expression among $\mathrm{CD} 7^{+} \mathrm{CD}^{+} \mathrm{T}$ cells identifies a CD8 $\mathrm{T}$ cell subset associated with viral control during chronic human immunodeficiency virus infection. J Virol. 2014;88(20):11861-11871.

91. Nguyen S, et al. Elite control of HIV is associated with distinct functional and transcriptional signatures in lymphoid tissue $\mathrm{CD}^{+} \mathrm{T}$ cells. Sci Transl Med. 2019;11(523):eaax4077.

92. Rutishauser RL, et al. TCF-1 regulates HIVspecific CD8 ${ }^{+} \mathrm{T}$ cell expansion capacity. JCI Insight. 2021;6(3):136648.

93. Gorin AM, et al. HIV-1 epitopes presented by MHC class I types associated with superior immune containment of viremia have highly constrained fitness landscapes. PLoS Pathog. 2017;13(8):e1006541.

94. Gaiha GD, et al. Structural topology defines protective $\mathrm{CD}^{+} \mathrm{T}$ cell epitopes in the HIV proteome. Science. 2019;364(6439):480-484.

95. Reuter MA, et al. HIV-specific CD8 ${ }^{+} \mathrm{T}$ cells exhibit reduced and differentially regulated cytolytic activity in lymphoid tissue. Cell Rep. 2017;21(12):3458-3470.

96. Buggert $M$, et al. Identification and characterization of HIV-specific resident memory $\mathrm{CD}^{+}$ T cells in human lymphoid tissue. Sci Immunol. 2018;3(24):eaar4526.

97. Kiniry BE, et al. Detection of HIV-1-specific gastrointestinal tissue resident $\mathrm{CD}^{+} \mathrm{T}$-cells in chronic infection. Mucosal Immunol. 2018;11(3):909-920.

98. Sáez-Cirión A, et al. Heterogeneity in HIV suppression by CD8 T cells from HIV controllers: association with Gag-specific CD8 T cell responses. JImmunol. 2009;182(12):7828-7837.

99. Ndhlovu ZM, et al. Elite controllers with low to absent effector CD8+ T cell responses maintain highly functional, broadly directed central memory responses. J Virol. 2012;86(12):6959-6969. 100.Schooley RT, et al. AIDS Clinical Trials Group
5197: a placebo-controlled trial of immunization of HIV-1-infected persons with a replication-deficient adenovirus type 5 vaccine expressing the HIV-1 core protein. JInfect Dis. 2010;202(5):705-716.

101.Samri A, et al. Polyfunctional HIV-specific T cells in post-treatment controllers. AIDS. 2016;30(15):2299-2302.

102. Ostrowski SR, et al. Residual viraemia in HIV-1-infected patients with plasma viral load. Scand J Immunol. 2008;68(6):652-660.

103. Hunt PW, et al. Relationship between T cell activation and $\mathrm{CD} 4^{+} \mathrm{T}$ cell count in HIV-seropositive individuals with undetectable plasma HIV RNA levels in the absence of therapy. JInfect Dis. 2008;197(1):126-133.

104.Pereyra F, et al. Persistent low-level viremia in HIV-1 elite controllers and relationship to immunologic parameters. JInfect Dis. 2009;200(6):984-990.

105.Liu Z, et al. CD8 ${ }^{+}$T-lymphocyte activation in HIV-1 disease reflects an aspect of pathogenesis distinct from viral burden and immunodeficiency. JAcquir Immune Defic Syndr Hum Retrovirol. 1998;18(4):332-340.

106. Giorgi JV, et al. Shorter survival in advanced human immunodeficiency virus type 1 infection is more closely associated with T lymphocyte activation than with plasma virus burden or virus chemokine coreceptor usage. J Infect Dis. 1999;179(4):859-870.

107. Kuller LH, et al. Inflammatory and coagulation biomarkers and mortality in patients with HIV infection. PLoS Med. 2008;5(10):e203.

108. Hazenberg MD, et al. Persistent immune activation in HIV-1 infection is associated with progression to AIDS. AIDS. 2003;17(13):1881-1888.

109. Pereyra F, et al. Increased coronary atherosclerosis and immune activation in HIV-1 elite controllers. AIDS. 2012;26(18):2409-2412.

110. Crowell TA, et al. Hospitalization rates and reasons among HIV elite controllers and persons with medically controlled HIV infection. J Infect Dis. 2015;211(11):1692-1702.

111. Brusca RM, et al. Subclinical cardiovascular disease in HIV controller and long-term nonprogressor populations. HIV Med. 2020;21(4):217-227.

112. Dominguez-Molina B, et al. Analysis of non-AIDS-defining events in HIV controllers. Clin Infect Dis. 2016;62(10):1304-1309.

113. Crowell TA, et al. Hospitalizations among HIV controllers and persons with medically controlled HIV in the U.S. Military HIV Natural History Study. J Int AIDS Soc. 2016;19(1):20524.

114. Tsimberidou AM, et al. Defining, identifying, and understanding "exceptional responders" in oncology using the tools of precision medicine. Cancer J. 2019;25(4):296-299.

115. Iyer $\mathrm{G}$, et al. Genome sequencing identifies a basis for everolimus sensitivity. Science. 2012;338(6104):221. 